\title{
La technologie peut aider à mieux prédire les chutes
}

\author{
Clemens Becker
}

Les technologies de l'information et de la communication comblent des lacunes qui ne peuvent pas être uniquement comblées avec les méthodes cliniques et les entretiens. Le dialogue est important entre les personnes spécialisées en gérontologie, en gériatrie avec les spécialistes en siences de l'ingénieur, en sciences du mouvement et en sciences de l'informatique afin de passer des démarches orientées vers les solutions à des démarches résolvant des problèmes.

\section{Analyse des chutes grâce à la vidéo}

Au cours des deux dernières années, des analyses de données vidéo ont été publiées pour la première fois. Elles montrent que notre compréhension des chutes des personnes âgées peut et doit être élargie à l'aide des procédés technologiques. L'une de ces études clé est le travail effectué par des ingénieurs à Vancouver (Robinovitch et al. 2013) qui ont pu enregistrer et exploiter des données à l'aide de plusieurs centaines de caméras et avec l'accord des pensionnaires de deux résidences médicalisées. Plus de 1000 chutes ont alors pu être analysées dans les couloirs et les salles communes. En raison des sites des caméras, les chutes ne sont pas représentatives mais apportent tout de même de nouveaux éclaircissements sur les mécanismes de chute qui n'auraient probablement pas pu être constatés sans ce procédé. Parmi les conclusions les plus importantes, l'analyse des problèmes de l'adaptation de la personne à son environnement occupe la première place. En particulier les problèmes d'application et les faiblesses/erreurs de conception des aides à la marche comme les déambulateurs ou les fauteuils roulants existent clairement. D'autres alternatives sont bien identifiables.

\section{Le savoir-faire gérontologique est questionné}

Ici le personnel spécialisé dans le domaine de la physiothérapie et de l'ergothérapie, formé en gérontologie, est invité à sortir des sentiers battus avec les ingénieurs et les concepteurs. Le site Internet du groupe TIPS (Technologies for Injury Prevention Studies, http://www.sfu.ca/tips) est recommandé à toute personne intéressée. En plus des problèmes d'adaptation de la personne à l'environnement, la deuxième conclusion importante est le nombre très élevé de chutes avec choc à la tête qui est bien plus élevé qu'on le supposait auparavant. Sur plus de 30\% des chutes filmées, on peut voir un net contact du crâne avec le sol. Ceci recoupe de nouvelles données épidémiologiques qui, même dans les pays où le taux de fracture de la hanche recule, montrent une augmentation nette des lésions cérébrales. Ceci soulève la question de la pose 
de l'indication des médicaments fluidifiant le sang chez les personnes présentant un risque de chute, question à laquelle on doit répondre de manière urgente puisque ces médicaments sont de plus en plus prescrits chez les personnes de grand âge également.

\section{Potentiel élevé de l'estimation des risques}

Un troisième domaine dans lequel se dessine un potentiel élevé est celui de l'estimation des risques de chute (prédiction) et, en étroite liaison, de la détection automatique des chutes. Ceci est lié à une amélioration des technologies de capteurs portables et à une amélioration des algorithmes lors de l'analyse des mouvements. Depuis environ trois ans, il est possible d'enregistrer les modèles de mouvements quotidiens sur plusieurs jours et au-delà non seulement les données d'accélération mais également les taux de rotation (signaux gyroscopiques). Ainsi, il est possible d'obtenir une meilleure analyse de l'orientation dans l'espace. Enfin, ce progrès a été rendu possible grâce à l'amélioration de la capacité de la batterie et de la mémoire. Depuis, des analyses ont été menées non pas uniquement dans les petits groupes de sujets mais également dans des échantillons représentatifs de la population. La prédiction des chutes avec évaluation clinique était jusqu'ici insuffisante avec des valeurs de $60 \%$ et moins. Parmi les personnes âgées questionnées sur leur chute, très peu peuvent donner une description de la chute (Yang et al. 2015). Grâce à une évaluation (non) supervisée et menée à l'aide de capteurs, la prédiction peut être sensiblement améliorée (van Schooten et al. 2015) Les mêmes démarches améliorent également l'identification et la description des chutes. Ceci revêt surtout de l'importance, car de nombreuses chutes n'ont pas lieu dans les zones sous vidéosurveillance mais dans les zones non surveillées. Ainsi des lacunes ont été comblées et ont permis d'améliorer les démarches préventives (Becker \& Chiari 2013). Les études montrent également l'importance de l'anamnèse et du questionnement des personnes présentant un risque de chute ou qui ont déjà connu une chute. La technologie complète alors le procédé clinique.

\section{Littérature :}

Becker, C. \& Chiari, L. (2013) What videos can tell us about falling. Lancet, 381(9860), 8-9.

Robinovitch, S.N. et al. (2013) Video capture of the circumstances of falls in elderly people residing in long-term care : an observational study. Lancet, 381(9860), 47-54.

van Schooten, K.S. et al. (2015) Ambulatory fall-risk assessment : amount and quality of daily-life gait predict falls in older adults. J Gerontol A Biol Sci Med Sci., 70(5), 608-615.

Yang, Y., Feldman, F., Leung, P.M., Scott, V. \& Robinovitch, S.N. (2015) Agreement between video footage and fall incident reports on the circumstances of falls in long-term care. J Am Med Dir Assoc., 16(5), 388-394. 
Auteur : Prof. Dr. Clemens Becker, Robert-Bosch-Krankenhaus, Division Gériatrie et clinique pour la réadaptation gériatrique, Stuttgart, Allemagne.

Contact : $\underline{\text { Clemens.Becker@rbk.de }}$ 